\title{
Society of Actuaries
}

Article from:

\section{North American Actuarial Journal}

\author{
Volume 13 - Number 2
}




\title{
A Robustification of the Chain-Ladder METHOD
}

\author{
Tim Verdonck, ${ }^{*}$ Martine Van Wouwe, ${ }^{\dagger}$ and Jan Dhaene ${ }^{\ddagger}$
}

\begin{abstract}
In a non-life insurance business an insurer often needs to build up a reserve to able to meet his or her future obligations arising from incurred but not reported completely claims. To forecast these claims reserves, a simple but generally accepted algorithm is the classical chain-ladder method. Recent research essentially focused on the underlying model for the claims reserves to come to appropriate bounds for the estimates of future claims reserves. Our research concentrates on scenarios with outlying data. On closer examination it is demonstrated that the forecasts for future claims reserves are very dependent on outlying observations. The paper focuses on two approaches to robustify the chain-ladder method: the first method detects and adjusts the outlying values, whereas the second method is based on a robust generalized linear model technique. In this way insurers will be able to find a reserve that is similar to the reserve they would have found if the data contained no outliers. Because the robust method flags the outliers, it is possible to examine these observations for further examination. For obtaining the corresponding standard errors the bootstrapping technique is applied. The robust chain-ladder method is applied to several run-off triangles with and without outliers, showing its excellent performance.
\end{abstract}

\section{INTRODUCTION}

Determining the expected profit or loss in a non-life insurance business is of growing importance because of the Solvency II regulations. This implies that an insurer has to be able to estimate the future claims reserves as accurately as possible. For an insurer operating in the non-life insurance business, the ultimate claims amount of an accident year is often not known at the end of that year. It will depend on the business line in the non-life insurance industry, and, for instance, in liability insurance it may be expected that the claims settlement will last several years because of bodily injuries and/or long-lasting trials. Also the possible time lag between the occurrence of the accident and the manifestation of the consequences of the event may cause the delay.

This leads to the consideration of a run-off triangle to organize the claims reserves. The goal of claims reserving is to estimate the outstanding claims reserve. One of the most popular methods to solve this problem is the classical chain-ladder method.

In this article we will focus on the appearance of outlying data in the run-off triangle of claims reserves. Looking for the cause of the high dependency of the chain-ladder estimate on outlying data brings us to robust statistics. Robust statistics gives the opportunity to develop a robust chain-ladder method that can recognize the outliers in the run-off triangle and that can smooth the outlying data in the run-off triangle in such a way that the outstanding claims reserve will be very close to the outstanding claims reserve without outliers. We will focus on two objectives: the first item is to develop

\footnotetext{
* Tim Verdonck is a PhD student in the Department of Mathematics and Computer Science, University of Antwerp, Belgium, tim.verdonck@ua.ac.be.

${ }^{\dagger}$ Martine Van Wouwe, PhD, is a Professor in the Faculty of Applied Economics, University of Antwerp, Belgium, martine.vanwouwe@ua.ac.be. ¥Jan Dhaene, PhD, is a Professor in the Faculty of Business and Economics, Katholieke Universiteit Leuven, Belgium, Jan.Dhaene@econ.kuleuven.be.
} 
a statistical technique to detect the outliers with a high probability, the second objective is to eliminate the influence of the outliers on the expected claims reserves by adjusting the classical chain-ladder method. A justification for the second goal is that insurers in this way will be able to measure the distance between the results for the expected claims reserves with and without outliers. If they produce different outcomes, a closer look at the data is recommended.

Since the estimates of the chain-ladder method can also be reproduced by using a generalized linear model and since there already exists an algorithm for obtaining robust estimates when using generalized linear models, this straightforward approach to obtain robust estimates for the outstanding claims reserve will also be studied.

Besides the reserve estimates, other aspects of the model are of importance. From a statistical viewpoint, given a point estimate, the natural next step is to estimate the likely variability in the outcomes. After estimating the variability, we can construct a confidence interval that is of great interest as the estimated claims reserve will never be an exact forecast of the future claims reserve. Another important measure in prediction problems is the root mean square error or the prediction error. For estimating the standard and prediction error of the different estimates we will apply the bootstrapping technique. We will explain for what reason we choose the bootstrapping technique for the standard error estimation, and we will therefore refer to the current debate on the standard error estimation of the classical chain-ladder method, which up to now is inconclusive. For the robust estimators we have modified the classical bootstrapping technique as suggested in Stromberg (1997).

Numerical results will show very satisfactory results for our robust chain-ladder method. We have also considered some run-off triangles from practice, from which it can be concluded that the robust method can cope with several outliers and that outliers do occur in practice.

In Section 2 we will give a short introduction to claims reserving, and in Section 3 the chain-ladder method will be explained. It will be demonstrated in Section 4 that the outstanding claims reserve is sensitive to outlying data. The robust version of the chain-ladder method and the robustification of the method based on generalized linear models will be described in Sections 5 and 6, respectively. The estimation of the standard and prediction errors with the bootstrapping technique will be explained in Section 7. The comparison of the different techniques will be made in Section 8, and real examples from practice will be studied in Section 9. The conclusions are given in Section 10.

\section{Claims Reserving}

The claims studied are the ones that are known to exist, but for which the eventual size is unknown at the time the reserves have to be set, also referred to as incurred but not reported completely (IBNRC) claims. The claims we concentrate on are the ones that take months or years to emerge, depending on the complexity of the damage. The delay in payment is, for example, due to long legal procedures or difficulties in determining the size of the claims. Therefore, insurers have to build up reserves enabling them to pay the outstanding claims and to meet claims arising in the future on the written contracts. We assume to have the following set of incremental claims:

$$
\left\{X_{i j} \mid i=1, \ldots, n ; j=1, \ldots, n-i+1\right\} .
$$

The past data are used to estimate the future claims amounts. The suffix $i$ refers to the row and could indicate accident year or underwriting year, whereas the suffix $j$ refers to the column and indicates the delay or development year. The claims on the diagonal with $i+j-1=c$ denote the payments that were made in calender year c. For example, $X_{42}$ is the payment that emerged in the second (after the fourth, i.e., the fifth) year for the claim originated in the fourth year. The techniques can easily be extended to semiannual, quarterly, or monthly data, but most insurance companies utilize annual data. In most cases it is irrelevant whether incremental or cumulative data are used. Often the two forms are needed, and it is very easy to convert from one to another. The cumulative claims are defined as

$$
C_{i j}=\sum_{k=1}^{j} X_{i k}
$$


Table 1

\section{Run-Off Triangle}

\begin{tabular}{|c|c|c|c|c|c|c|c|}
\hline Development Year & & & & & & & \\
\cline { 1 - 5 } Accident Year & $\mathbf{1}$ & $\mathbf{2}$ & $\ldots$ & $\boldsymbol{n}-\boldsymbol{i}+\mathbf{1}$ & $\ldots$ & $\boldsymbol{n}-\mathbf{1}$ & $\boldsymbol{n}$ \\
\hline 1 & $X_{11}$ & $X_{12}$ & $\ldots$ & $X_{1, n-i+1}$ & $\ldots$ & $X_{1, n-1}$ & $X_{1 n}$ \\
2 & $X_{21}$ & $X_{22}$ & $\ldots$ & $X_{2, n-i+1}$ & $\ldots$ & $X_{2, n-1}$ & \\
$\vdots$ & $\ldots$ & $\ldots$ & $\ldots$ & $X_{i, n-i+1}$ & & & \\
$i$ & $X_{i 1}$ & $X_{i 2}$ & $\ldots$ & & & \\
$\vdots$ & $\ldots$ & $\ldots$ & $\ldots$ & & & & \\
\hline
\end{tabular}

with the values of $C_{i j}$ for $i+j \leq n+1$ known. We are interested in the values of $C_{i j}$ for $i+j>n+$ 1 , in particular the ultimate claims amounts $C_{i n}$ of each accident year $i=2, \ldots, n$. The outstanding claims reserve of accident year $i$ is defined as

$$
R_{i}=C_{i n}-C_{i, n-i+1}, \quad 1 \leq i \leq n,
$$

taking into account that $C_{i, n-i+1}$ has already been paid. The insurance company is, of course, interested in the overall reserve $R$, being the sum of the reserves $R_{i}$ for $i=1, \ldots, n$. For representation of the data it is common to use a run-off triangle as in Table 1.

The aim of claims reserving is to make predictions about claims that will be paid in future calender years on the basis of the claims figures in the run-off triangle. Therefore the ultimate goal of a claims reserving method is to complete the triangle into a square, because the total of the values found in the lower right triangle equals the overall reserve $R$ that will have to be paid in the future. Various claims reserving methods exist that are based on different assumptions and/or meeting specific requirements. In the next section we will describe one of the oldest and most popular techniques for estimating outstanding claims reserves, namely, the chain-ladder method.

\section{Chain-Ladder Method}

The chain-ladder method is based on the assumption that the expectations underlying the columns and the rows in the run-off triangle are proportional. This straightforward method has been heavily critized. However, several authors have countered the criticism by constructing stochastic models that support the chain-ladder method. In practice it seems rational to use the chain-ladder method to estimate the outstanding claims reserve if the data are consistent with the model. The chainladder method uses cumulative data and assumes the existence of a set of development factors $\left\{f_{j} \mid j=\right.$ $2, \ldots, n\}$, with

$$
\mathrm{E}\left[C_{i, k+1} \mid C_{i 1}, \ldots, C_{i n}\right]=C_{i k} f_{k+1}, \quad 1 \leq i \leq n, 1 \leq k \leq n-1 .
$$

These factors are estimated by the chain-ladder method as

$$
\hat{f}_{j}=\frac{\sum_{i=1}^{n-j+1} C_{i j}}{\sum_{i=1}^{n-j+1} C_{i, j-1}}, \quad 2 \leq j \leq n .
$$

To forecast future values of cumulative claims, these factors are applied to the latest cumulative claim in each row:

$$
\begin{aligned}
\hat{C}_{i, n-i+2} & =C_{i, n-i+1} \hat{f}_{n-i+2}, & & 2 \leq i \leq n \\
\hat{C}_{i, k} & =\hat{C}_{i, k-1} \hat{f}_{k}, & & 2 \leq i \leq n, \quad n-i+3 \leq k \leq n .
\end{aligned}
$$

As already stated in the introduction, it is also important to estimate the reserve variability. Mack (1993, 1994) has derived a distribution-free formula for the standard error of chain-ladder reserve estimates. In this article we will not study the theoretical variability or standard error of the estimates, 
but we will estimate it in a consistent way by using the bootstrapping technique. The main reason for this choice is that the actuarial literature remains undecided about which assumptions finally support the chain-ladder method (Mack et al. 2006; Venter 2006; Wüthrich et al. 2008). We will focus on this item in more detail when the evaluation of the standard error is discussed in Section 7.

\section{Sensitivity of the Chain-Ladder Method toward Outlying Values}

When analyzing real data, it may occur that one or more observations differ from the majority. Such observations are called outliers. Sometimes they are due to recording or copying mistakes (for example, a misplaced decimal point). Often the outlying observations are not incorrect, but they were made under exceptional circumstances and consequently do not fit the model well. In these situations, robust statisties tries to find a fit that is similar to the fit we would have found without the outlier(s). In practice it is also very important to be able to detect these outliers for further examination. In this regard it is important to note that outlying values are defined by the distribution of the majority of the data. When data are resulting from a long-tailed distribution, large values are not necessarily outlying because they may fit the underlying distribution.

We are interested to know how the chain-ladder method reacts toward outliers. By looking at a very simple example, it will be clear that the presence of outlying claims may lead to incorrect reserve estimates.

The run-off triangle in Table 2 satisfies completely the assumptions of the chain-ladder method because the ratios between the columns are the same for each row. We will study the effect of multiplying a single observation by a constant by calculating the outstanding reserve estimate with the chainladder method before and after the addition of the outlier.

The expected future claims estimates obtained with the classical chain-ladder method on the uncontaminated run-off triangle (in Table 2) are given in Table 3. By summing up all expected future claims we get an overall reserve $R=7,482.5$.

Table 2

Run-Off Triangle with Same Ratio between

Columns for Each Row

\begin{tabular}{|c|c|c|c|c|c|c|}
\hline & $\mathbf{1}$ & $\mathbf{2}$ & $\mathbf{3}$ & $\mathbf{4}$ & $\mathbf{5}$ & $\mathbf{6}$ \\
\hline 1 & 12,000 & 6,000 & 600 & 300 & 150.0 & 15.00 \\
2 & 13,000 & 6,500 & 650 & 325 & 162.5 & $\cdot$ \\
3 & 10,000 & 5,000 & 500 & 250 & $\cdot$ & $\cdot$ \\
4 & 12,000 & 6,000 & 600 & $\cdot$ &. & $\cdot$ \\
5 & 11,000 & 5,500 &. &. &. &. \\
6 & 10,000 &. &. &. &. &. \\
\hline
\end{tabular}

Table 3

Future Claims Estimates, Obtained with Chain-Ladder Method

\begin{tabular}{|c|c|c|c|c|c|c|}
\hline & $\mathbf{1}$ & $\mathbf{2}$ & $\mathbf{3}$ & $\mathbf{4}$ & $\mathbf{5}$ & $\mathbf{6}$ \\
\hline 1 & 12,000 & 6,000 & 600 & 300 & 150.0 & 15.00 \\
2 & 13,000 & 6,500 & 650 & 325 & 162.5 & 16.25 \\
3 & 10,000 & 5,000 & 500 & 250 & 125.0 & 12.50 \\
4 & 12,000 & 6,000 & 600 & 300 & 150.0 & 15.00 \\
5 & 11,000 & 5,500 & 550 & 275 & 137.5 & 13.75 \\
6 & 10,000 & 5,000 & 500 & 250 & 125.0 & 12.50 \\
\hline
\end{tabular}


Table 4

Future Claims Estimates, Obtained with Chain-Ladder Method

\begin{tabular}{|c|c|r|l|l|r|r|}
\hline & $\mathbf{1}$ & $\mathbf{2}$ & \multicolumn{1}{|c|}{$\mathbf{3}$} & \multicolumn{1}{|c|}{$\mathbf{4}$} & $\mathbf{5}$ & $\mathbf{6}$ \\
\hline 1 & 12,000 & $\mathbf{6 0 , 0 0 0}$ & 600 & 300 & 150.0 & 15.00 \\
2 & 13,000 & 6,500 & 650 & 325 & 162.5 & 4.24 \\
3 & 10,000 & 5,000 & 500 & 250 & 52.71 & 3.24 \\
4 & 12,000 & 6,000 & 600 & 150.35 & 62.75 & 3.86 \\
5 & 11,000 & 5,500 & 311.45 & 135.89 & 56.72 & 3.49 \\
6 & 10,000 & 14,310 & 458.87 & 200.21 & 83.57 & 5.14 \\
\hline
\end{tabular}

Note: Claim $X_{12}$ is made outlying by multiplication by 10 .

An outlying value was introduced to this run-off triangle by multiplying the claims amount $X_{12}$ by 10. Applying the chain-ladder method on this adjusted run-off triangle results in the estimated claims amounts of Table 4.

This leads to an overall reserve of $R=15,842.49$, which is more than twice the reserve $(7,482.5)$ obtained for the original run-off triangle (Table 2). The observation in the lower left corner is predicted to be much larger, whereas all the other claims are estimated as too low. Hence one outlier can cause a totally different reserving scheme for an insurance company using the classical chain-ladder method.

This suggests that the chain-ladder method is not robust, and therefore we will introduce in Section 5 a possible approach to make the chain-ladder method less sensitive to outliers. A robust version of the chain-ladder method will have a robust calculation of both the development factors and the claims amounts.

\section{The Robust Chain-Ladder Method}

This section presents a robust chain-ladder method. Our objective is not to replace the classical chainladder method by the proposed robust version, but it will certainly be very useful to apply both methods (the classical and the robust version) to the data and compare the overall reserve estimates. If both estimates are approximately the same, there is no problem, but when both versions give a different result, we recommend having a closer look at the data. The robust method indicates the presence of outlying claim(s) so that one can search for the reason(s) behind the atypical value(s).

In Section 5.1 we propose a robust way to calculate development factors, and in Section 5.2 we explain how outlying claims can be detected and adjusted.

\subsection{Robustification of the Development Factors}

The first step is to detect what causes the classical chain-ladder method to be so dependent on outlying data. The definition of the development factors is based on the cumulative data $C_{i j}$, and hence an outlier in the first column will affect all development factors. On the other hand, when working with the incremental data $X_{i j}$, an outlier can at most affect two development factors.

Instead of dividing the sum of one column by the sum of the previous column, we could also look at the ratios of the columns for each row:

$$
\left\{\frac{X_{i j}}{X_{i, j-1}} \mid i=1, \ldots, n-j+1 ; j=2, \ldots, n\right\} .
$$

By taking the mean of the ratios of the same columns, we could assume to get approximately the same development factors as defined in (3.1).

Hampel et al. (1986) have illustrated that the mean (by using the influence function) as a statistical tool is very sensitive toward outlying data. In examining the expression for the development factors, these factors can be viewed as a mean, which explains the dependency of the traditional chain-ladder 
Table 5

\section{Future Claims Estimates, Obtained with Robust} Chain-Ladder Method

\begin{tabular}{|c|c|c|c|c|c|c|}
\hline & $\mathbf{1}$ & $\mathbf{2}$ & $\mathbf{3}$ & $\mathbf{4}$ & $\mathbf{5}$ & $\mathbf{6}$ \\
\hline 1 & 12,000 & $\mathbf{6 0 , 0 0 0}$ & 600 & 300 & 150.0 & 15.00 \\
2 & 13,000 & 6,500 & 650 & 325 & 162.5 & 16.25 \\
3 & 10,000 & 5,000 & 500 & 250 & 125.0 & 12.50 \\
4 & 12,000 & 6,000 & 600 & 300 & 150.0 & 15.00 \\
5 & 11,000 & 5,500 & 550 & 275 & 137.5 & 13.75 \\
6 & 10,000 & 5,000 & 500 & 250 & 125.0 & 12.50 \\
\hline
\end{tabular}

Note: Claim $X_{12}$ is made outlying by multiplication by 10 .

method on outlying data. To solve this problem we have to replace the mean by a more robust estimate. The robust statistical literature (see Huber 1981; Hampel et al. 1986; Rousseeuw and Leroy 1987; Maronna et al. 2006) solved this problem by replacing the mean by the median, which is a more robust estimate. The median of an univariate data set is defined as the middle value (or the mean of the two values in the middle if there is no single point in the middle) of the ordered observations. Unlike the mean, the median is not influenced by outliers.

We therefore propose to use the median (in combination with incremental data), which leads to the following alternative definition of development factors:

$$
\tilde{f}_{j}=\operatorname{median}\left\{\frac{X_{i j}}{X_{i, j-1}} \mid i=1, \ldots, n-j+1\right\}, \quad 2 \leq j \leq n .
$$

Applying the proposed robust method to the run-off triangle of the previous section (see Table 2, contaminated with the same outlier $X_{12}$ ) results in the claims estimates of Table 5. The adjusted development factors seem to work perfectly, because they lead to the same future claims estimates as the one obtained with the classical chain-ladder method applied to the data without outliers.

Unfortunately some problems remain. For instance, an outlying value in the second last column will, even with these adjusted development factors, influence the estimated reserves. In this situation we only have two ratios, and taking the median is the same as taking the mean. Moreover, in that column we only have two claims amounts, which makes it very hard to decide which claim is outlying. In this example the outlying value is not used to calculate other claims estimates, and hence robustifying only the development factors is sufficient. However, if we would take the outlying value on the second last row, adjusting only the development factors will not work, as can be concluded from the results in Table 6.

The reason for the failure in this situation is that the outlier is still used to calculate the other claims estimates. Because solely robustifying the development factors is not sufficient, we have implemented a mechanism to detect and adjust the outlying values, which will be discussed in Section 5.2.

Table 6

Future Claims Estimates, Obtained with Chain-Ladder Method

\begin{tabular}{|c|c|c|c|c|c|c|}
\hline & $\mathbf{1}$ & $\mathbf{2}$ & $\mathbf{3}$ & $\mathbf{4}$ & $\mathbf{5}$ & $\mathbf{6}$ \\
\hline 1 & 12,000 & 6,000 & 600 & 300 & 150.0 & 15.00 \\
2 & 13,000 & 6,500 & 650 & 325 & 162.5 & 16.25 \\
3 & 10,000 & 5,000 & 500 & 250 & 125.0 & 12.50 \\
4 & 12,000 & 6,000 & 600 & 300 & 150.0 & 15.00 \\
5 & 11,000 & $\mathbf{5 5 , 0 0 0}$ & 2,200 & 1,100 & 550.0 & 55.00 \\
6 & 10,000 & $13,534.48$ & 784.48 & 392.24 & 196.12 & 19.61 \\
\hline
\end{tabular}

Note: Claim $X_{52}$ is made outlying by multiplication by 10 . 


\subsection{Detecting and Adjusting Outliers in the Chain-Ladder Method}

Our technique for detecting outlying observations in a run-off triangle of claims amounts consists of several steps. We will describe each step in this section. A concise summary can be found in Table 7 .

For the given formulas, we restrict ourselves to the model described by Renshaw and Verrall (1998), who proposed modeling the incremental claims using an "overdispersed" Poisson distribution (hence the variance is proportional to the mean).

Step 1: Under the given restrictions it holds that (see England and Verrall 1999), for $1 \leq i \leq n$, $1 \leq j \leq n-i+1$ :

$$
\begin{aligned}
\mathrm{E}\left[X_{i j}\right] & =m_{i j}, \\
\operatorname{Var}\left[X_{i j}\right] & =\phi m_{i j},
\end{aligned}
$$

leading to the Pearson residuals, defined as

$$
r_{i j}=\frac{X_{i j}-m_{i j}}{\sqrt{\phi m_{i j}}},
$$

where $X$ are the incremental data, $\phi$ is a scale parameter, and $m$ are the incremental fitted values.

The development factors are calculated as in (5.1), but based on the observed cumulative data. As already mentioned in Section 5.1, the development factors in incremental form are more robust, but the approach we follow in this step (see England and Verrall 1999) works only with cumulative data. Because the fitted cumulative paid to date equals the actual cumulative paid to date, the final diagonal of the actual cumulative triangle can be transferred to the fitted cumulative triangle. The remaining cumulative fitted values are obtained backwards by recursively dividing the cumulative fitted value at time $t$ by the development factor at time $t-1$. The incremental fitted data $m$ are obtained by differencing the cumulative fitted values as described by England and Verrall (1999). By doing so, the cornerpoints at location $(1, n)$ and $(n, 1)$ of the triangle always equal the cornerpoints of the observed incremental data, and, therefore, the corresponding residuals will be zero.

Step 2: To detect outliers the classical boxplot is used, which was introduced by Tukey (1977). We considered some real and simulated triangles and note that residuals of outlying claims are more likely to lie outside the classical boxplot interval

$$
\left[Q_{1}-3 I Q R, Q_{3}+3 I Q R\right],
$$

where $Q_{1}$ and $Q_{3}$ are, respectively, the first and the third quartile. The classical boxplot rejection rule inherently assumes normality of the data, which seems to hold under the restrictions of our simulation study. Moreover, only a small number of residuals are available, which tend tests to accept normality due to low power values in small samples. If the assumption of normality is not satisfied (which can be tested with, for example, the Shapiro-Wilk test), one can use the adjusted boxplot (Hubert and Vandervieren 2008) when the residuals are skewed. When the contaminated observations are realizations of a heavy-tailed payment distribution, the elimination might produce a downward bias. In this case, it is advisable to measure the tail weight in a robust manner (Brys et al. 2006) and apply a bias correction.

Step 3: When detecting an outlying residual in the first column, the corresponding claims amount is supposed to be outlying and will be altered. If the claims amount in the next column in the same row is also detected as an outlying value, the claim in the first column is replaced by the median of the claims of the first column. (Depending on the data it might be better to replace the claim by the mean of the claim above it and under it - if these are not outlying - which will probably take inflation better into account.)

In the other case, we divide the claims amount in the next column of the same row by a robust development factor, and we replace the outlying claim by this value. We do not use these residuals to investigate the claims in the other columns, because the used development factors are based on cumulative data (which is necessary to obtain the fitted data as in England and Verrall 1999), and, therefore, one outlying claim can affect more than just its corresponding residual. 
Step 4: To detect possible outliers in the other columns, we will switch again to residuals, but the fitted data are obtained in a different manner. From the previous steps we know that the first column has already become outlier free. Therefore, the development factors are now calculated based on the incremental claims of the first column:

$$
\hat{f}_{j}^{1}=\operatorname{median}\left\{\frac{X_{i j}}{X_{i 1}} \mid i=1, \ldots, n-j+1\right\}, \quad 2 \leq j \leq n .
$$

Consequently the future claims are estimated as

$$
\hat{X}_{i j}^{1}=X_{i 1} \hat{f}_{j}^{1}, \quad 2 \leq i \leq n, n-i+2 \leq j \leq n .
$$

We fit the upper triangle by multiplying the claims of the first column with these development factors, so that a new set of incremental claims is created. The corresponding residuals are calculated as in (5.2), using the estimates $\hat{X}_{i j}^{1}$ as the incremental fitted values (denoted as $m_{i j}$ ). By doing so, an outlying claim (almost) affects only its corresponding residual. All residuals of the first column equal zero, and therefore possible outliers in the first column cannot be detected using these residuals.

\section{Table 7}

\section{Different Steps of Proposed Technique}

\section{Step 1}

- Compute development factors

$$
\tilde{f}_{j}=\operatorname{median}\left\{\frac{C_{i j}}{C_{i, j-1}} \mid i=1, \ldots, n-j+1\right\} \quad 2 \leq j \leq n .
$$

- Obtain Pearson residuals, $r_{i j}$ as in England and Verrall (1999).

Step 2

- Test for normality.

- Apply classical boxplot rejection rule on residuals.

Step 3

- If outlier in first column; suppose $r_{k 1}$

- If $r_{k 2}$ is not outlying,

$$
C_{k 1}=\frac{X_{k 2}}{\operatorname{median}\left\{\frac{X_{i 2}}{X_{i 1}} \mid i=1, \ldots, n-1\right\}} \quad 2 \leq k \leq n .
$$

- If $r_{k 2}$ is outlying,

$$
C_{k 1}=\operatorname{median}\left\{C_{i 1} \mid i=1, \ldots, n\right\}
$$

Step 4

- Compute development factors

$$
\hat{f}_{j}^{1}=\operatorname{median}\left\{\frac{X_{i j}}{X_{i, 1}} \mid i=1, \ldots, n-j+1\right\} \quad 2 \leq j \leq n .
$$

- Calculate fitted incremental claims

$$
\hat{X}_{j, n-i+2}^{1}=X_{j, 1} \hat{f}_{n-i+2}^{1} \quad 1 \leq j \leq n, 2 \leq i \leq n .
$$

- Obtain residuals, $r_{i j}^{1}$ as in (5.2).

- Apply classical boxplot rejection rule (after testing for normality).

- If outlier; suppose $r_{k l}^{1}$

$-r_{k l}^{l}=\operatorname{median}\left\{r_{i j}^{1} \mid i=1, \ldots, n-1 ; j=2, \ldots, n-i+1\right\}$.

- Backtransform residuals $r_{i j}^{1}$ to data matrix $X_{i j}^{r}$.

Step 5

- Apply classical chain-ladder method on the robustified data $X_{i j}^{r}$. 
The remaining residuals can be examined by using the boxplot. The outlying residuals are replaced by the median of the residuals. The final observations (denoted by $X_{i j}^{r}$ ) are obtained by backtransforming.

Step 5: Finally, the classical chain-ladder method is applied on the robustified data $X_{i j}^{r}$. Table 7 summarizes the different steps of our proposed technique. Note that with this proposed robust chainladder method the outlyingness of the claims in the cornerpoints, $X_{1 n}$ and $X_{n 1}$, cannot be investigated.

$X_{n 1}$ is the first payment of the claim originated in the last accident year, and hence it is hard to say whether this value is atypical. As a possible solution we suggest taking the median of values of the first column and verifying whether this value differs much from $X_{n 1}$ (for this the boxplot interval can also be used). $X_{1 n}$, on the other hand, is the only claim where development year $n$ is considered, and so we have no idea whether the corresponding value is atypical or not. Here we suggest extrapolating the last development factor by using a curve estimation model based on the former factors and verifying whether this value differs significantly from the last development factor estimated using claim $X_{1 n}$. We explored some possible statistical curve estimation models on several real run-off triangles. From these results, it appears that the inverse model with equation $\tilde{f}_{j}=b_{0}+b_{1} 1 / j(2 \leq j \leq n)$ preliminarily often gives good results, but, of course, the choice for the best model is data dependent. For more information we refer to Van Wouwe et al. (2009).

Also, for the two claims of the last but one column, this approach can certainly be implemented, because it is possible to extrapolate the last but one development factor based on the former factors. Hence we can see whether the two ratios in that column differ much from the predicted last but one development factor. If only one of the ratios is detected as atypical, the other ratio will be taken as the corresponding development factor. If both ratios are outlying, the fitted development factor (obtained by extrapolation) will be chosen. Recall that the median over both ratios forms the last but one development factor. If one of these two ratios is outlying, the corresponding development factor will be influenced. Therefore the approach based on the curve estimation models might give better results.

In Section 8 we will show how the proposed robust method performs on the data set provided in Taylor and Ashe (1983) when an outlier is introduced. In that example the model $\tilde{f}_{j}=b_{0}+b_{1} e^{-j}$ $(2 \leq j \leq n)$ yielded a very nice fit and was chosen as optimal curve estimation model. In Section 9 some real run-off triangles will be analyzed with the classical and robust chain-ladder method. In these examples the inverse model was always used as the curve estimation model.

\section{The Robust GLM Method}

In recent years considerable attention has been given to discuss possible relationships between the chain-ladder and various stochastic models (see, e.g., Mack 1993, 1994; Verrall 1991, 2000; Renshaw and Verrall 1998; England and Verrall 1999; Mack and Venter 2000). Several stochastic models used for claims reserving can be embedded within the framework of generalized linear models (GLMs), introduced by Nelder and Wedderburn (1972). England and Verrall (2002) provide a review of stochastic reserving models for claims reserving based on GLMs. The textbook Modern Actuarial Risk Theory (Kaas et al. 2001) also presents claims reserving models in the framework of GLMs.

Let us consider the multiplicative model

$$
X_{i j} \approx \alpha_{i} \beta_{j} \gamma_{k}
$$

which has a parameter for each row $i$, each column $j$, and each diagonal $k=i+j-1$. When the random variables $X_{i j}$ are independent and we restrict their distribution to be in the exponential dispersion family, (6.1) is a GLM. The expected value of $X_{i j}$ is the exponent of the linear form $\log \alpha_{i}+$ $\log \beta_{j}+\log \gamma_{i+j-1}$, so that there is a logarithmic link. The chain-ladder method can be derived from (6.1) if the following assumption about the distributions is satisfied:

$$
X_{i j} \sim \operatorname{Poisson}\left(\alpha_{i} \beta_{j}\right) \quad \text { independent; } \gamma_{k} \equiv 1 \text {. }
$$

When calculating the parameters $\alpha_{i}>0$ and $\beta_{j}>0$ by maximum likelihood estimation, we obtain a multiplicative GLM with Poisson errors and a log-link. The optimal estimates of the parameters $\alpha_{i}$ and 
$\beta_{j}$ produced by this GLM are identical to the parameter estimates found by the chain-ladder method (this still holds for the overdispersed Poisson model). Note that the proposed method is not the only one that is consistent with the chain-ladder method. Other stochastic models that can be expressed within the framework of GLM and give exactly the same forecasts as the chain-ladder method are, for example, the overdispersed negative binomial model and Mack's model.

\subsection{Robust Estimators for GLM}

The nonrobustness of the maximum likelihood estimator (and the usual quasi-likelihood estimator) for the model parameters $\beta$ has already been studied by several authors, including Pregibon (1982), Stefanski et al. (1986), Künsch et al. (1989), Morgenthaler (1992), and Carroll and Pederson (1993).

Preisser and Qaqish (1999) proposed a class of robust estimators in the generalized estimating equations framework of Liang and Zeger (1986). Starting from this class of robust estimators, Cantoni and Ronchetti (2001) proposed a set of robust inferential tools that apply to the whole class of GLMs and are based on a natural generalization of the quasi-likelihood approach. They considered a general class of $\mathrm{M}$ estimators of Mallows's type, where the influence functions of deviations on the response and on the predictors are bounded separately.

For the estimation of binomial and Poisson models the code for this method can be downloaded as part of a robust library (namely, the library "robustbase") in the statistical software program R (http: //www.r-project.org). Since the reserve estimates of the chain-ladder method can be obtained by using a maximum likelihood estimation in a model with independent Poisson $\left(\alpha_{i} \beta_{j}\right)$ variables $X_{i j}$, and since a robust method for fitting GLM is already available, we will also study this straightforward way to obtain robust estimates.

\section{Estimation of the Standard and Prediction Errors}

In addition to the reserve estimates, it is important in practice to obtain the standard errors; hence the precision of the estimates can be calculated (for example, by constructing confidence intervals). How to estimate the standard error for the classical chain-ladder method is already a point of discussion. We refer to the current debate (Mack et al. 2006; Venter 2006; Wütrich et al. 2008) that there is no unique answer to which assumptions support the chain-ladder method. Several articles have introduced stochastic models with different assumptions leading to the same mean estimate but with different distributions for the standard error estimate. The ongoing discussion is focused on the set of assumptions, but till now the actuarial world has been inconclusive.

Estimates of the standard error can also be used to obtain an estimate for the root mean square error of prediction (also known as the prediction error), which is often used in prediction problems. Renshaw (1994) used first-degree Taylor expansions to deduce an approximation for the mean square error of prediction for the individual predictions $\left(E\left[\left(X_{i j}-\hat{X}_{i j}\right)^{2}\right]\right)$, for the row totals $\left(E\left[\left(R_{i}-\hat{R}_{j}\right)^{2}\right]\right)$, and for the total reserve $\left(E\left[(R-\hat{R})^{2}\right]\right)$ for the log-normal, overdispersed Poisson, and Gamma reserving models (see England and Verrall 1999 for more information). However, those estimates are very difficult to calculate and still remain approximations.

Where a standard (or prediction) error is difficult to estimate analytically, it is common to adopt the bootstrapping technique, which will be explained in Section 7.1.

\subsection{Bootstrap Technique}

A popular method that produces a simulated predictive distribution for obtaining the standard errors of well-specified models is bootstrapping (see Efron and Tibshirani 1993 for an introduction). The bootstrapping technique has already been considered in the field of claims reserving (Ashe 1986; Lowe 1994; Pinheiro et al. 2003; England and Verrall 2006; Barnett and Zehnwirth 2008) and has proven to be a very convenient tool. In the context of claims reserving it is common to bootstrap the residuals rather than the data themselves because of the dependency between some observations and the param- 
eter estimates. Recall that the resampling is based on the hypothesis that the residuals are independent and identically distributed. Within the framework of GLMs different types of residuals can be chosen, but it is common to use Pearson residuals as defined in (5.2) for Poisson GLMs. As already mentioned, the residuals in the cornerpoints at location $(1, n)$ and $(n, 1)$ are equal to zero (which is also noticed in England 2002 and Pinheiro et al. 2003). We therefore decided not to use the residuals corresponding to the last column of the first row and to the first row of the last column in the resampling procedure of the bootstrapping technique.

The statistical literature indicates that the determination of the distribution of the standard error for a robust estimator is an even more complex problem. In a few cases an attempt has been undertaken to come to an answer (see, for example, Croux et al. 2003). A more global solution for robust estimators is still a big issue in robust statistics. These observations made us decide to also apply the bootstrapping technique for evaluating the standard error for our robust chain-ladder estimation of the outstanding claims reserve.

As shown in Stromberg (1997), the bootstrapped sample covariance matrix can have a breakdown point of $1 / n$ regardless of the robustness of the original estimate. Hence the breakdown value is approximately zero for large $n$. The breakdown value is defined as the smallest proportion of observations in the data set that need to be replaced to carry the estimate arbitrarily far away. Therefore bootstrapped covariance estimates may be heavily influenced by outliers even if the original estimate is not. In Stromberg (1997) it is concluded that by estimating the variance of robust estimators in a reliable way the bootstrapping technique can be applied, but instead of using the sample variance in the last step of the computation of the bootstrap variance estimate, a more robust measure should be used. The robust measure of scale we considered is the median absolute deviation (MAD), given by the median of all absolute distances from the sample median:

$$
\text { MAD }=1.483 \operatorname{median}_{j=1, \ldots, n}\left|x_{j}-\operatorname{median}_{i=1, \ldots, n}\left(x_{i}\right)\right|,
$$

where the constant 1.483 is a correction factor that makes the MAD unbiased for the normal distribution. The breakdown value of the MAD is $50 \%$, whereas the sample variance has breakdown point zero.

After acquiring the bootstrap standard error of the reserve estimate (denoted as $S E_{b s}(R)$ ), the prediction error of the total reserve (denoted as $P E_{b s}(R)$ ) can be obtained. England and Verrall (1999) suggest a bias correction because the variance of the residuals is smaller than the variance of the underlying random variable. Moreover, the variance of each residual depends not only on the random variable, but also on the data structure of the model. The bootstrap standard error of prediction with bias correction can be computed as

$$
P E_{b s}(R)=\sqrt{\phi_{p} R+\frac{n}{n-p}\left(S E_{b s}(R)\right)^{2}},
$$

where an estimate of the Pearson scale parameter $\left(\phi_{p}\right)$ is given by

$$
\phi_{p}=\frac{\Sigma r^{2}}{n-p},
$$

with $n$ the number of observations in the data triangle and $p$ the number of parameters estimated. The summation is over the number $(n)$ of residuals.

\section{Comparison of the Different Claims Reserving Methods}

In this section we will consider the data from Taylor and Ashe (1983) and compare the different methods, namely,

- clasCL: the classical chain-ladder method, described in Section 3

- robCL: the robust chain-ladder method, described in Section 5 
- clasGLM: the very basic GLM method with independent Poisson variables

- robGLM: the same as clasGLM, but with the parameters estimated by the robust algorithm of Cantoni and Ronchetti (2001), described in Section 6.1.

This data set, which is presented in incremental form in Table 8 , has already been used by many authors (see, for example, Mack 1993; Renshaw 1994; England and Verrall 1999; Pinheiro et al. 2000).

An outlying value is added by multiplying a claims amount by 10 . We have chosen to multiply by 10 because this comes down to misplacing the decimal point one step to the right (which might be human error). Since the influence of an outlier is strongly dependent on its location, it is appropriate to look at each observation separately.

In Table 9 the estimated reserves for the different methods are presented. The first line represents the results obtained for the original dataset (without outliers), for which the forecasts for the future claims reserves for all methods should coincide. We immediately see that the chain-ladder method, its robust version, and the method based on GLM indeed give exactly the same result for the outstanding claims reserve. The robust method based on GLM also performs well. We can conclude that it is safe to use the robust versions on data without outliers.

For the following lines, the first column shows which claim was multiplied by 10 (and hence can be considered as an outlier). As expected, the classical chain-ladder method and the classical method based on GLM give exactly the same results. It can be concluded that both classical methods cannot handle a single outlier and that the influence of the outlier on the estimated claims reserve depends much on the location of that outlier. We also see that, although most of the time the claims reserve gets overestimated, sometimes an underestimation also occurs, even though all outliers were created by multiplication by 10 .

Surprisingly the robust version of the GLM method is also significantly influenced by the atypical observation included in the data. We would like to note that this method always warned that the algorithm did not converge, but nowhere was it explained how this could be solved. On the other hand, the results of the robust chain-ladder method are very satisfactory. The average number of detected observations equals 1.27, and the method always succeeded in detecting the added outlier. Because we always adjusted exactly one observation of the data, we can conclude that the robust chain-ladder method performs very well.

The good properties of the robust chain-ladder method can also be demonstrated graphically: in Figure 1 we have plotted the reserve estimates for the different methods and the different data sets (each with a different outlier, in the same order as in Table 9). From this it is very clear that one outlier can have an enormous effect on the estimated outstanding reserves obtained by the classical methods (and by the robust GLM method). The robust chain-ladder method, on the other hand, always finds a result very near the true outstanding reserve $(18,680,856)$, defined as the estimate for the data set without the outlier (marked on the plot with the dashed line).

Table 8

Claims Data from Taylor and Ashe (1983)

\begin{tabular}{|c|c|c|c|c|c|c|c|c|c|c|}
\hline & 1 & 2 & 3 & 4 & 5 & 6 & 7 & 8 & 9 & 10 \\
\hline $\begin{array}{r}1 \\
2 \\
3 \\
4 \\
5 \\
6 \\
7 \\
8 \\
9 \\
10\end{array}$ & $\begin{array}{l}357,848 \\
352,118 \\
290,507 \\
310,608 \\
443,160 \\
396,132 \\
440,832 \\
359,480 \\
376,686 \\
344,014\end{array}$ & $\begin{array}{r}766,940 \\
884,021 \\
1,001,799 \\
1,108,250 \\
693,190 \\
937,085 \\
847,631 \\
1,061,648 \\
986,608\end{array}$ & $\begin{array}{r}610,542 \\
933,894 \\
926,219 \\
776,189 \\
991,983 \\
847,498 \\
1,131,398 \\
1,443,370\end{array}$ & $\begin{array}{r}482,940 \\
1,183,289 \\
1,016,654 \\
1,562,400 \\
769,488 \\
805,037 \\
1,063,269\end{array}$ & $\begin{array}{l}527,326 \\
445,745 \\
750,816 \\
272,482 \\
504,851 \\
705,960\end{array}$ & $\begin{array}{l}574,398 \\
320,996 \\
146,923 \\
352,053 \\
470,639\end{array}$ & $\begin{array}{l}146,342 \\
527,804 \\
495,992 \\
206,286\end{array}$ & $\begin{array}{l}139,950 \\
266,172 \\
280,405\end{array}$ & $\begin{array}{l}227,229 \\
425,046\end{array}$ & 67,948 \\
\hline
\end{tabular}


Table 9

\section{Estimated Reserves for the Different Methods}

\begin{tabular}{|c|c|c|c|c|}
\hline outl & clasCL & robCL & clasGLM & robGLM \\
\hline- & $18,680,856$ & $18,680,856$ & $18,680,856$ & $18,839,333$ \\
\hline$x_{11}$ & $12,603,783$ & $18,487,959$ & $12,603,783$ & $13,328,668$ \\
\hline$X_{12}$ & $12,851,784$ & $18,411,731$ & $12,851,784$ & $13,041,001$ \\
\hline$X_{13}$ & $15,813,130$ & $18,370,569$ & $15,813,130$ & $15,908,930$ \\
\hline$X_{14}$ & $18,169,959$ & $18,419,406$ & $18,169,959$ & $18,270,311$ \\
\hline$X_{15}$ & $2,013,275$ & $18,681,093$ & $20,132,751$ & $19,847,842$ \\
\hline$X_{16}$ & $22,709,179$ & $18,584,734$ & $22,709,179$ & $22,139,912$ \\
\hline$X_{17}$ & $20,329,847$ & $18,879,228$ & $20,329,847$ & $20,185,642$ \\
\hline$X_{18}$ & $21,616,873$ & $19,149,029$ & $21,616,873$ & $21,204,448$ \\
\hline$X_{19}$ & $28,068,004$ & $18,700,368$ & $28,068,004$ & $27,677,539$ \\
\hline$X_{1,10}$ & $26,382,875$ & $20,266,192$ & $26,382,875$ & $26,621,669$ \\
\hline$X_{21}$ & $13,064,239$ & $18,619,218$ & $13,064,239$ & $13,536,364$ \\
\hline$x_{22}$ & $13,080,595$ & $18,628,484$ & $13,080,595$ & $13,087,107$ \\
\hline$X_{23}$ & $16,044,692$ & $18,713,353$ & $16,044,692$ & $15,934,573$ \\
\hline$X_{24}$ & $20,313,528$ & $18,757,158$ & $20,313,528$ & $19,691,394$ \\
\hline$X_{25}$ & $20,318,298$ & $18,713,874$ & $20,318,298$ & $20,091,626$ \\
\hline$x_{26}$ & $21,142,008$ & $18,740,127$ & $21,142,008$ & $20,980,352$ \\
\hline$x_{27}$ & $25,833,401$ & $18,526,323$ & $25,833,401$ & $25,321,955$ \\
\hline$X_{28}$ & $24,770,914$ & $18,865,708$ & $24,770,914$ & $24,618,051$ \\
\hline$X_{29}$ & $36,975,225$ & $17,788,537$ & $36,975,225$ & $37,819,011$ \\
\hline$X_{31}$ & $14,594,660$ & $16,911,913$ & $14,594,660$ & $15,119,785$ \\
\hline$x_{32}$ & $14,881,519$ & $18,942,881$ & $14,881,519$ & $14,840,586$ \\
\hline$X_{33}$ & $18,190,072$ & $17,856,414$ & $18,190,072$ & $17,907,853$ \\
\hline$X_{34}$ & $22,260,738$ & $18,608,427$ & $22,260,738$ & $21,398,414$ \\
\hline$X_{35}$ & $23,910,232$ & $18,437,900$ & $23,910,232$ & $23,031,220$ \\
\hline$X_{36}$ & $20,281,482$ & $18,899,668$ & $20,281,482$ & $20,445,844$ \\
\hline$x_{37}$ & $26,993,251$ & $18,345,131$ & $26,993,251$ & $26,217,904$ \\
\hline$X_{38}$ & $26,124,080$ & $18,501,515$ & $26,124,080$ & $25,530,928$ \\
\hline$X_{41}$ & $14,890,583$ & $18,344,006$ & $14,890,583$ & $15,635,844$ \\
\hline$X_{42}$ & $16,119,238$ & $18,927,113$ & $16,119,238$ & $16,109,039$ \\
\hline$X_{43}$ & $19,166,515$ & $18,677,034$ & $19,166,515$ & $18,992,655$ \\
\hline$X_{44}$ & $26,779,838$ & $18,260,491$ & $26,779,838$ & $26,004,658$ \\
\hline$X_{45}$ & $20,950,173$ & $18,892,896$ & $20,950,173$ & $20,737,414$ \\
\hline$X_{46}$ & $23,178,356$ & $18,649,556$ & $23,178,356$ & $22,780,256$ \\
\hline$X_{47}$ & $22,505,925$ & $18,950,015$ & $22,505,925$ & $22,044,677$ \\
\hline$X_{51}$ & $14,864,015$ & $19,021,397$ & $14,864,015$ & $15,396,659$ \\
\hline$X_{52}$ & $17,682,939$ & $18,483,164$ & $17,682,939$ & $17,790,906$ \\
\hline$X_{53}$ & $21,424,577$ & $18,706,467$ & $21,424,577$ & $21,077,167$ \\
\hline$X_{54}$ & $23,930,482$ & $19,199,746$ & $23,930,482$ & $23,297,747$ \\
\hline$X_{55}$ & $24,047,427$ & $18,934,684$ & $24,047,427$ & $23,316,993$ \\
\hline$X_{56}$ & $25,775,382$ & $18,733,871$ & $25,775,382$ & $24,961,291$ \\
\hline$X_{61}$ & $15,906,579$ & $18,362,703$ & $15,906,579$ & $16,422,207$ \\
\hline$X_{62}$ & $19,712,830$ & $18,663,571$ & $19,712,830$ & $19,538,605$ \\
\hline$X_{63}$ & $22,724,309$ & $18,761,396$ & $22,724,309$ & $22,362,176$ \\
\hline$X_{64}$ & $26,011,071$ & $19,204,133$ & $26,011,071$ & $25,272,745$ \\
\hline$X_{65}$ & $27,889,017$ & $18,444,105$ & $27,889,017$ & $26,949,513$ \\
\hline$X_{71}$ & $17,251,335$ & $18,791,335$ & $17,251,335$ & $17,487,130$ \\
\hline$x_{72}$ & $22,268,233$ & $18,761,392$ & $22,268,233$ & $21,978,585$ \\
\hline$X_{73}$ & $28,111,881$ & $18,605,367$ & $28,111,881$ & $27,487,874$ \\
\hline$X_{74}$ & $32,254,649$ & $19,273,972$ & $32,254,649$ & $31,182,740$ \\
\hline$X_{81}$ & $20,451,046$ & $18,679,791$ & $20,451,046$ & $20,305,126$ \\
\hline$X_{82}$ & $32,812,737$ & $18,491,335$ & $32,812,737$ & $31,926,498$ \\
\hline$X_{83}$ & $44,155,256$ & $17,673,888$ & $44,155,256$ & $43,304,970$ \\
\hline$X_{91}$ & $27,531,995$ & $18,643,601$ & $27,531,995$ & $27,787,925$ \\
\hline$X_{92}$ & $50,350,360$ & $18,336,128$ & $50,350,360$ & $50,669,456$ \\
\hline & $60,313,152$ & $19,004,501$ & $60,313,152$ & $61,101,893$ \\
\hline
\end{tabular}

Note: Each claim is made outlying by multiplication by 10 .

The corresponding standard errors $S E_{b s}(R)$ for the different methods can be found in Table 10. From the first line (which represents the situation without outlier), we see that the robust chain-ladder method has a higher standard error than the other methods. This phenomenon does not arrive unexpectedly, but is simply the result of the well-known trade-off between robustness and efficiency. The higher standard deviations (in the uncontaminated case) are the price we need to pay for making 
Figure 1

\section{Plot of Reserve Estimates for the Different Methods}

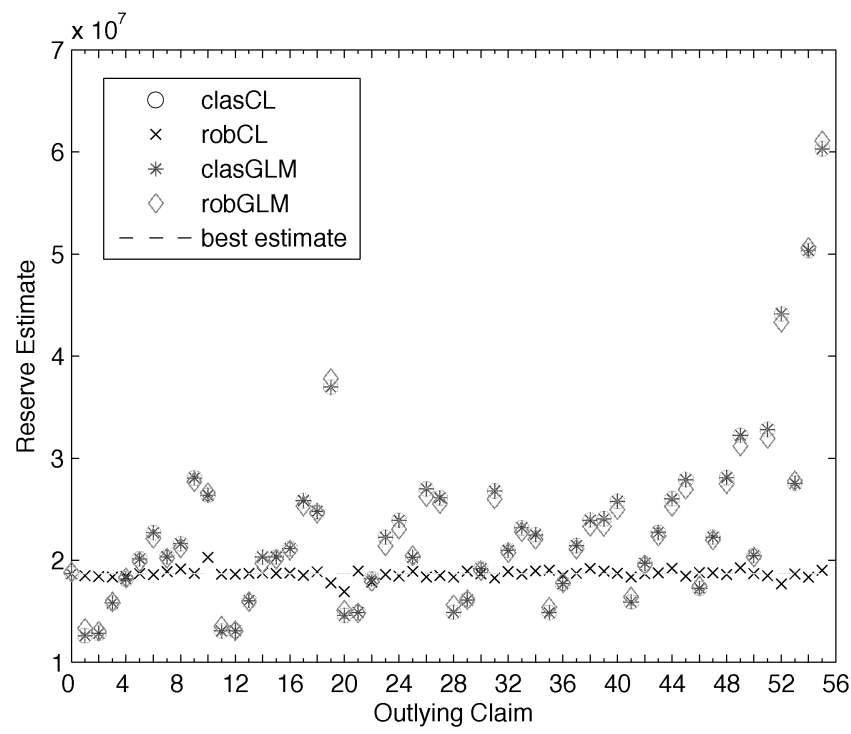

the method robust. As soon as there are outliers in the data we see that the robustification is worth its price, because then the robust chain-ladder method has (nearly) always the lowest standard error. The results are visualized in Figure 2. We will not look at the prediction errors, because these will lead to analogous conclusions.

Note that we do not define how many outliers the proposed robust method resists or give other theoretical results, but it is obvious that the robust method breaks down when there are more outlying than regular claims in a certain column. For the rows the number of outliers is not crucial.

\section{Real Run-Off Triangles from Practice}

In this section we will discuss the results of applying classical and robust chain-ladder methods on real run-off triangles. We have studied several data sets from a non-life business line of the Belgian insurance industry, but in this article we will focus on two examples. Because of the results of Section 8 we will compare only the classical and the robust chain-ladder method. In the first run-off triangle (see Table 11) there is only a small difference between both methods.

For the classical chain-ladder method the estimated future claims (with estimated reserves for the rows) are shown in Table 12. The classical method suggests putting aside an overall reserve $R=$ $\sum_{i=1}^{10} R_{i}=1,463,388,937$. On the other hand, the robust chain-ladder method indicates one outlier in the data, which is situated at the upper right corner, namely, claim $X_{2,9}(=24,602,209)$. The robust chain-ladder method adjusted this claims amount into the value 18,408,361. We now get $f_{9}=1.037351$. The estimated future claims can be found in Table 13.

The outstanding reserve estimated with the robust chain-ladder method equals 1,437,093,149, which is close to the classical outstanding reserve estimate. In this example it does not make much difference whether claim $X_{2,9}$ is altered or not. Taking a closer look at the data, we see that the flagged observation is a bit abnormal, but to classify it as a clear outlier is questionable. This explains partly the small difference between the classical and the robust outstanding reserve estimate. It is advisable for the insurance company to have a good look at the claims amount $X_{3,9}$, which will be known in the following year.

In the second example (the run-off triangle is shown in Table 14) we see a totally different situation. Applying the classical chain-ladder method on the data of Table 14 results in the estimated future 
Table 10

Standard Errors for the Different Methods

\begin{tabular}{|c|c|c|c|c|}
\hline outl & clasCL & robCL & clasGLM & robGLM \\
\hline - & $1,599,681$ & $2,190,625$ & $1,599,681$ & $1,604,451$ \\
\hline$X_{11}$ & $2,707,399$ & $2,138,764$ & $2,707,399$ & $2,340,320$ \\
\hline$X_{12}$ & $2,508,137$ & $2,203,704$ & $2,508,137$ & $2,340,814$ \\
\hline$x_{13}$ & $3,121,649$ & $2,350,954$ & $3,121,649$ & $2,844,233$ \\
\hline$X_{14}$ & $2,841,367$ & $2,261,444$ & $2,841,367$ & $2,493,747$ \\
\hline$X_{15}$ & $3,904,724$ & $2,320,335$ & $3,904,724$ & $3,733,201$ \\
\hline$X_{16}$ & $4,688,239$ & $2,048,458$ & $4,688,239$ & $4,281,581$ \\
\hline$X_{17}$ & $2,237,214$ & $2,208,538$ & $2,237,214$ & $2,129,203$ \\
\hline$X_{18}$ & $2,465,573$ & $2,338,262$ & $2,465,573$ & $2,448,975$ \\
\hline$X_{19}$ & $3,171,971$ & $2,037,788$ & $3,171,971$ & $3,060,583$ \\
\hline$X_{1,10}$ & $2,075,936$ & $2,159,432$ & $2,075,936$ & $2,047,558$ \\
\hline$X_{21}$ & $2,378,233$ & $2,366,410$ & $2,378,233$ & $2,022,685$ \\
\hline$x_{22}$ & $2,529,390$ & $2,258,192$ & $2,529,390$ & $2,449,173$ \\
\hline$X_{23}$ & $3,520,701$ & $2,215,397$ & $3,520,701$ & $3,217,143$ \\
\hline$X_{24}$ & $4,510,697$ & $2,365,810$ & $4,510,697$ & $4,199,791$ \\
\hline$X_{25}$ & $3,342,324$ & $2,216,542$ & $3,342,324$ & $3,128,617$ \\
\hline$X_{26}$ & $2,815,376$ & $2,340,805$ & $2,815,376$ & $2,763,902$ \\
\hline$x_{27}^{2}$ & $4,317,741$ & $2,157,039$ & $4,317,741$ & $4,357,442$ \\
\hline$X_{28}$ & $3,166,066$ & $2,407,339$ & $3,166,066$ & $2,973,168$ \\
\hline$X_{29}$ & $4,482,630$ & $1,948,994$ & $4,482,630$ & $4,228,221$ \\
\hline$x_{31}$ & $2,350,920$ & $1,855,530$ & $2,350,920$ & $2,178,909$ \\
\hline$x_{32}$ & $3,321,491$ & $2,609,415$ & $3,321,491$ & $3,113,798$ \\
\hline$X_{33}$ & $3,955,689$ & $1,994,907$ & $3,955,689$ & $3,550,845$ \\
\hline$X_{34}$ & $4,797,606$ & $2,423,098$ & $4,797,606$ & $4,349,172$ \\
\hline$X_{35}$ & $4,958,183$ & $2,172,776$ & $4,958,183$ & $4,544,714$ \\
\hline$X_{36}$ & $1,901,238$ & $2,203,838$ & $1,901,238$ & $1,840,326$ \\
\hline$x_{37}$ & $4,628,842$ & $2,214,219$ & $4,628,842$ & $4,473,625$ \\
\hline$X_{38}$ & $3,421,691$ & $2,281,821$ & $3,421,691$ & $3,273,970$ \\
\hline$X_{41}$ & $2,725,833$ & $2,500,379$ & $2,725,833$ & $2,327,929$ \\
\hline$X_{42}$ & $3,668,152$ & $2,624,998$ & $3,668,152$ & $3,438,979$ \\
\hline$X_{43}$ & $3,905,010$ & $2,347,477$ & $3,905,010$ & $3,609,447$ \\
\hline$X_{44}$ & $6,847,643$ & $2,085,716$ & $6,847,643$ & $6,243,778$ \\
\hline$X_{45}$ & $2,468,426$ & $2,080,671$ & $2,468,426$ & $2,381,012$ \\
\hline$X_{46}$ & $3,378,777$ & $2,381,872$ & $3,378,777$ & $3,209,046$ \\
\hline$X_{47}$ & $2,580,219$ & $2,171,184$ & $2,580,219$ & $2,301,840$ \\
\hline$X_{51}$ & $3,533,580$ & $2,436,801$ & $3,533,580$ & $3,125,872$ \\
\hline$X_{52}$ & $3,298,418$ & $2,063,795$ & $3,298,418$ & $3,263,016$ \\
\hline$X_{53}$ & $4,741,418$ & $2,420,305$ & $4,741,418$ & $4,658,461$ \\
\hline$X_{54}$ & $4,365,858$ & $2,402,410$ & $4,365,858$ & $4,196,580$ \\
\hline$X_{55}$ & $4,247,732$ & $2,545,286$ & $4,247,732$ & $3,978,034$ \\
\hline$X_{56}$ & $4,725,018$ & $2,397,985$ & $4,725,018$ & $4,351,699$ \\
\hline$X_{61}$ & $2,933,998$ & $2,129,124$ & $2,933,998$ & $2,741,726$ \\
\hline$x_{62}$ & $4,040,140$ & $2,300,819$ & $4,040,140$ & $3,707,742$ \\
\hline$X_{63}$ & $4,594,332$ & $2,357,594$ & $4,594,332$ & $4,176,063$ \\
\hline$X_{64}$ & $4,424,659$ & $2,373,618$ & $4,424,659$ & $4,345,120$ \\
\hline$X_{65}$ & $5,359,747$ & $2,139,368$ & $5,359,747$ & $4,999,749$ \\
\hline$X_{71}$ & $3,399,221$ & $2,329,294$ & $3,399,221$ & $2,943,646$ \\
\hline$x_{72}$ & $3,766,183$ & $2,171,800$ & $3,766,183$ & $3,377,502$ \\
\hline$x_{73}$ & $5,356,830$ & $2,149,406$ & $5,356,830$ & $5,164,167$ \\
\hline$X_{74}$ & $5,754,261$ & $2,417,282$ & $5,754,261$ & $5,671,909$ \\
\hline$X_{81}$ & $2,971,165$ & $2,278,977$ & $2,971,165$ & $2,924,589$ \\
\hline$X_{82}$ & $4,988,374$ & $2,409,523$ & $4,988,374$ & $4,973,210$ \\
\hline$X_{83}$ & $7,330,020$ & $2,032,515$ & $7,330,020$ & $7,117,901$ \\
\hline$X_{91}$ & $3,894,843$ & $2,321,417$ & $3,894,843$ & $3,754,685$ \\
\hline$X_{92}$ & $6,170,230$ & $2,334,134$ & $6,170,230$ & $5,978,958$ \\
\hline$X_{10,1}$ & $5,667,162$ & $2,308,422$ & $5,667,162$ & $5,516,394$ \\
\hline
\end{tabular}

Note: The claim is made outlying by multiplication by 10 .

claims and outstanding reserves for the rows of Table 15. From the classical chain-ladder method it follows that the insurance company has to set aside a reserve $R=18,673,307$.

The robust chain-ladder method indicates seven outliers in the run-off triangle of Table 16 . The outlying observations coincide completely with the third row of the run-off triangle (except the first observation $X_{31}$ ). The claims originated in the third accident year are indeed exceptionally high, which 
Figure 2

\section{Plot of Estimates of Standard Errors for the Different Methods}

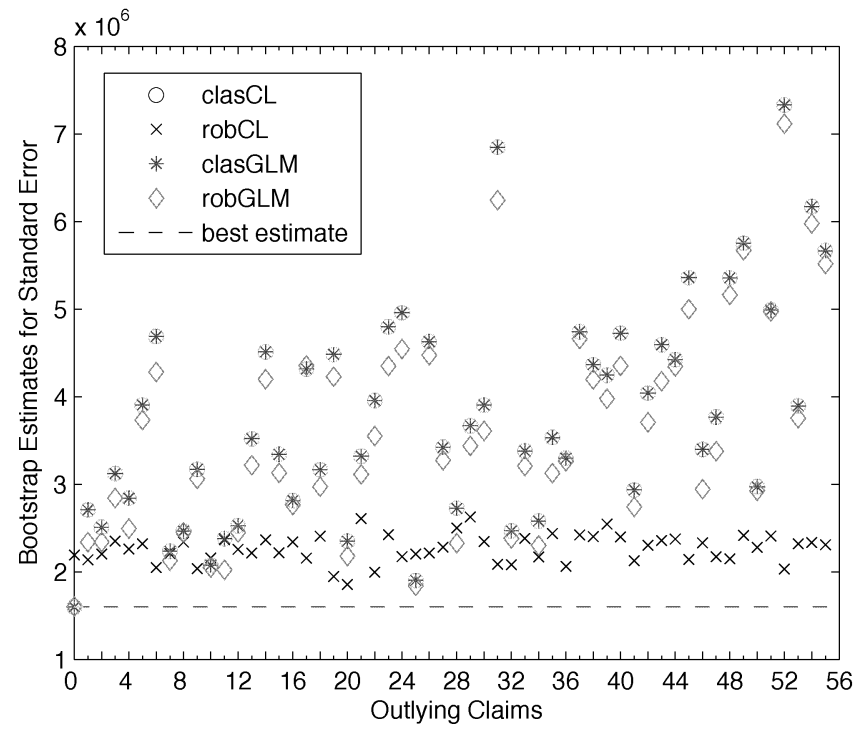

Table 11

Real Run-Off Triangle from Practice (Example 1)

\begin{tabular}{|c|c|c|c|c|c|c|c|c|c|c|}
\hline & 1 & 2 & 3 & 4 & 5 & 6 & 7 & 8 & 9 & 10 \\
\hline 1 & $135,338,126$ & $90,806,681$ & $68,666,715$ & $55,736,215$ & $46,967,279$ & $35,463,367$ & $30,477,244$ & $24,838,121$ & $18,238,489$ & $14,695,083$ \\
\hline 2 & $125,222,434$ & $89,639,978$ & $70,697,962$ & $58,649,114$ & $46,314,227$ & $41,369,299$ & $34,394,512$ & $26,554,172$ & $24,602,209$ & \\
\hline 3 & $136,001,521$ & $91,672,958$ & $78,246,269$ & $62,305,193$ & $49,115,673$ & $36,631,598$ & $30,210,729$ & $29,882,359$ & & \\
\hline 4 & $135,277,744$ & $103,604,885$ & $78,303,084$ & $61,812,683$ & $48,720,135$ & $39,271,861$ & $32,029,697$ & & & \\
\hline 5 & $143,540,778$ & $109,316,613$ & $79,092,473$ & $65,603,900$ & $51,226,270$ & $44,408,236$ & & & & \\
\hline 6 & $132,095,863$ & $88,862,933$ & $69,269,383$ & $57,109,637$ & $48,818,781$ & & & & & \\
\hline 7 & $127,299,710$ & $92,979,311$ & $61,379,607$ & $50,317,305$ & & & & & & \\
\hline 8 & $120,660,241$ & $89,469,673$ & $71,570,718$ & & & & & & & \\
\hline 9 & $134,132,283$ & $87,016,365$ & & & & & & & & \\
\hline 10 & $131,918,566$ & & & & & & & & & \\
\hline
\end{tabular}

Table 12

Example 1, Completed with the Classical Chain-Ladder Method

\begin{tabular}{|c|c|c|c|c|c|c|c|c|c|c|c|}
\hline & 1 & 2 & 3 & 4 & 5 & 6 & 7 & 8 & 9 & 10 & $R_{i}$ \\
\hline 1 & $135,338,126$ & $90,806,681$ & $68,666,715$ & $55,736,215$ & $46,967,279$ & $35,463,367$ & $30,477,244$ & $24,838,121$ & $18,238,489$ & $14,695,083$ & 0 \\
\hline 2 & $125,222,434$ & $89,639,978$ & $70,697,962$ & $58,649,114$ & $46,314,227$ & $41,369,299$ & $34,394,512$ & $26,554,172$ & $24,602,209$ & $15,011,643$ & $15,011,643$ \\
\hline 3 & $136,001,521$ & $91,672,958$ & $78,246,269$ & $62,305,193$ & $49,115,673$ & $36,631,598$ & $30,210,729$ & $29,882,359$ & $22,446,400$ & $15,564,850$ & $38,011,250$ \\
\hline 4 & $135,277,744$ & $103,604,885$ & $78,303,084$ & $61,812,683$ & $48,720,135$ & $39,271,861$ & $32,029,697$ & $28,684,424$ & $23,041,905$ & $15,977,787$ & $67,704,115$ \\
\hline 5 & $143,540,778$ & $109,316,613$ & $79,092,473$ & $65,603,900$ & $51,226,270$ & $44,408,236$ & $35,104,160$ & $30,367,042$ & $24,393,535$ & $16,915,038$ & $106,779,775$ \\
\hline 6 & $132,095,863$ & $88,862,933$ & $69,269,383$ & $57,109,637$ & $48,818,781$ & $37,514,208$ & $30,867,825$ & $26,702,378$ & $21,449,747$ & $14,873,748$ & $131,407,907$ \\
\hline 7 & $127,299,710$ & $92,979,311$ & $61,379,607$ & $50,317,305$ & $44,199,591$ & $35,622,093$ & $29,310,935$ & $25,355,582$ & $20,367,880$ & $14,123,557$ & $168,979,637$ \\
\hline 8 & $120,660,241$ & $89,469,673$ & $71,570,718$ & $55,012,848$ & $44,830,352$ & $36,130,447$ & $29,729,224$ & $25,717,425$ & $20,658,545$ & $14,325,110$ & $226,403,951$ \\
\hline 9 & $134,132,283$ & $87,016,365$ & $70,456,750$ & $56,947,133$ & $46,406,615$ & $37,400,815$ & $30,774,522$ & $26,621,665$ & $21,384,912$ & $14,828,790$ & $304,821,202$ \\
\hline \multirow[t]{2}{*}{10} & $131,918,566$ & $93,526,403$ & $71,825,534$ & $58,053,462$ & $47,308,170$ & $38,127,412$ & $31,372,388$ & $27,138,852$ & $21,800,363$ & $15,116,873$ & $404,269,457$ \\
\hline & & & & & & & & & & & $1,463,388,937$ \\
\hline
\end{tabular}


Table 13

Example 1, Completed with the Robust Chain-Ladder Method

\begin{tabular}{|c|c|c|c|c|c|c|c|c|c|c|c|}
\hline & 1 & 2 & 3 & 4 & 5 & 6 & 7 & 8 & 9 & 10 & $R_{i}$ \\
\hline 1 & $135,338,126$ & $90,806,681$ & $68,666,715$ & $55,736,215$ & $46,967,279$ & $35,463,367$ & $30,477,244$ & $24,838,121$ & $18,238,489$ & $14,695,083$ & 0 \\
\hline 2 & $125,222,434$ & $89,639,978$ & $70,697,962$ & $58,649,114$ & $46,314,227$ & $41,369,299$ & $34,394,512$ & $26,554,172$ & $18,408,361$ & $14,831,952$ & $14,831,952$ \\
\hline 3 & $136,001,521$ & $91,672,958$ & $78,246,269$ & $62,305,193$ & $49,115,673$ & $36,631,598$ & $30,210,729$ & $29,882,359$ & $19,201,131$ & $15,470,701$ & $34,671,833$ \\
\hline 4 & $135,277,744$ & $103,604,885$ & $78,303,084$ & $61,812,683$ & $48,720,135$ & $39,271,861$ & $32,029,697$ & $28,684,424$ & $19,710,539$ & $15,881,140$ & $64,276,103$ \\
\hline 5 & $143,540,778$ & $109,316,613$ & $79,092,473$ & $65,603,900$ & $51,226,270$ & $44,408,236$ & $35,104,160$ & $30,367,042$ & $20,866,752$ & $16,812,722$ & $103,150,676$ \\
\hline 6 & $132,095,863$ & $88,862,933$ & $69,269,383$ & $57,109,637$ & $48,818,781$ & $37,514,208$ & $30,867,825$ & $26,702,378$ & $18,348,573$ & $14,783,780$ & $128,216,764$ \\
\hline 7 & $127,299,710$ & $92,979,311$ & $61,379,607$ & $50,317,305$ & $44,199,591$ & $35,622,093$ & $29,310,935$ & $25,355,582$ & $17,423,121$ & $14,038,126$ & $165,949,447$ \\
\hline 8 & $120,660,241$ & $89,469,673$ & $71,570,718$ & $55,012,848$ & $44,830,352$ & $36,130,447$ & $29,729,224$ & $25,717,425$ & $17,671,762$ & $14,238,460$ & $223,330,518$ \\
\hline 9 & $134,132,283$ & $87,016,365$ & $70,456,750$ & $56,947,133$ & $46,406,615$ & $37,400,815$ & $30,774,522$ & $26,621,665$ & $18,293,111$ & $14,739,093$ & $301,639,705$ \\
\hline \multirow[t]{2}{*}{10} & $131,918,566$ & $93,526,403$ & $71,825,534$ & $58,053,462$ & $47,308,170$ & $38,127,412$ & $31,372,388$ & $27,138,852$ & $18,648,497$ & $15,025,434$ & $401,026,153$ \\
\hline & & & & & & & & & & & $1,437,093,149$ \\
\hline
\end{tabular}

Table 14

Real Run-Off Triangle from Practice (Example 2)

\begin{tabular}{|c|c|c|c|c|c|c|c|c|c|c|}
\hline & 1 & 2 & 3 & 4 & 5 & 6 & 7 & 8 & 9 & 10 \\
\hline $\begin{array}{l}1 \\
2 \\
3 \\
4 \\
5 \\
6 \\
7 \\
8 \\
9\end{array}$ & $\begin{array}{r}701,848 \\
1,864,592 \\
11,52,332 \\
966,722 \\
789,602 \\
1,154,888 \\
1,053,622 \\
1,956,875 \\
1,568,152\end{array}$ & $\begin{array}{r}232,585 \\
856,348 \\
2,381,638 \\
168,570 \\
485,170 \\
475,018 \\
459,830 \\
368,372 \\
966,498\end{array}$ & $\begin{array}{r}194,470 \\
441,065 \\
2,545,868 \\
149,128 \\
192,082 \\
619,605 \\
419,665 \\
244,525\end{array}$ & $\begin{array}{r}148,488 \\
256,385 \\
2,613,448 \\
140,050 \\
149,400 \\
330,220 \\
273,385\end{array}$ & $\begin{array}{r}98,600 \\
139,112 \\
2,310,415 \\
38,410 \\
140,052 \\
91,025\end{array}$ & $\begin{array}{r}61,875 \\
108,032 \\
2,712,015 \\
9,548 \\
43,518\end{array}$ & $\begin{array}{r}47,145 \\
62,855 \\
3,662,850 \\
12,308\end{array}$ & $\begin{array}{r}32,260 \\
47,355 \\
3,704,750\end{array}$ & $\begin{array}{l}25,628 \\
33,132\end{array}$ & 18,173 \\
\hline
\end{tabular}

Table 15

Example 2, Completed with Classical Chain-Ladder Method

\begin{tabular}{|c|c|c|c|c|c|c|c|c|c|c|c|}
\hline & 1 & 2 & 3 & 4 & 5 & 6 & 7 & 8 & 9 & 10 & $R_{i}$ \\
\hline 1 & 701,848 & 232,585 & 194,470 & 148,488 & 98,600 & 61,875 & 47,145 & 32,260 & 25,628 & 18,173 & 0 \\
\hline 2 & $1,864,592$ & 856,348 & 441,065 & 256,385 & 139,112 & 103,032 & 62,855 & 47,355 & 33,132 & 44,804 & 44,804 \\
\hline 3 & $1,152,332$ & $2,381,638$ & $2,545,868$ & $2,613,448$ & $2,310,415$ & $2,712,015$ & $3,662,850$ & $3,704,750$ & 234,276 & 251,089 & 485,365 \\
\hline 4 & 966,722 & 168,570 & 149,128 & 140,050 & 38,410 & 9,548 & 12,308 & 248,762 & 19,262 & 20,645 & 288,670 \\
\hline 5 & 789,602 & 485,170 & 192,082 & 149,400 & 140,052 & 43,518 & 335,820 & 357,819 & 27,707 & 29,696 & 751,042 \\
\hline 6 & $1,154,888$ & 475,018 & 619,605 & 330,220 & 91,025 & 408,495 & 574,541 & 612,180 & 47,403 & 50,805 & $1,693,424$ \\
\hline 7 & $1,053,622$ & 459,830 & 419,665 & 273,385 & 327,050 & 387,509 & 545,025 & 580,730 & 44,968 & 48,195 & $1,933,478$ \\
\hline 8 & $1,956,875$ & 368,372 & 244,525 & 580,847 & 466,988 & 553,317 & 778,230 & 829,213 & 64,209 & 68,817 & $3,341,620$ \\
\hline 9 & $1,568,152$ & 966,498 & 808,505 & 755,655 & 607,530 & 719,840 & $1,012,442$ & $1,078,768$ & 83,533 & 89,527 & $5,155,799$ \\
\hline \multirow[t]{2}{*}{10} & $1,322,485$ & 754,419 & 662,493 & 619,187 & 497,813 & 589,840 & 829,600 & 883,947 & 68,447 & 73,359 & $4,979,105$ \\
\hline & & & & & & & & & & & $18,673,307$ \\
\hline
\end{tabular}

Table 16

Example 2, Completed with Robust Chain-Ladder Method

\begin{tabular}{|r|r|c|c|c|r|r|r|r|r|r|r|}
\hline & \multicolumn{1}{|c|}{$\mathbf{1}$} & $\mathbf{2}$ & $\mathbf{3}$ & $\mathbf{4}$ & $\mathbf{5}$ & $\mathbf{6}$ & $\mathbf{7}$ & $\mathbf{8}$ & $\mathbf{9}$ & $\mathbf{1 0}$ & $\boldsymbol{R}_{\boldsymbol{i}}$ \\
\hline 1 & 701,848 & 232,585 & 194,470 & 148,488 & 98,600 & 61,875 & 47,145 & 32,260 & 25,628 & 18,173 & \\
2 & $1,864,592$ & 856,348 & 441,065 & 256,385 & 139,112 & 103,032 & 62,855 & 47,355 & 33,132 & 44,804 & 44,804 \\
3 & $1,152,332$ & 502,910 & 299,806 & 243,796 & 126,355 & 63,675 & 58,125 & 52,966 & 27,779 & 29,773 & 57,552 \\
4 & 966,722 & 168,570 & 149,128 & 140,050 & 38,410 & 9,548 & 12,308 & 25,714 & 16,784 & 17,988 & 60,486 \\
5 & 789,602 & 485,170 & 192,082 & 149,400 & 140,052 & 43,518 & 36,245 & 31,798 & 20,756 & 22,245 & 111,044 \\
6 & $1,154,888$ & 475,018 & 619,605 & 330,220 & 91,025 & 71,790 & 55,230 & 48,454 & 31,627 & 33,897 & 240,998 \\
7 & $1,053,622$ & 459,830 & 419,665 & 273,385 & 111,700 & 62,314 & 47,940 & 42,058 & 27,452 & 29,422 & 320,886 \\
8 & $1,956,875$ & 368,372 & 244,525 & 300,601 & 145,308 & 81,062 & 62,363 & 54,711 & 35,712 & 38,275 & 718,031 \\
9 & $1,568,152$ & 966,498 & 492,034 & 354,049 & 171,144 & 95,475 & 73,451 & 64,439 & 42,061 & 45,080 & $1,337,734$ \\
10 & $1,322,485$ & 532,752 & 360,145 & 259,146 & 125,269 & 69,883 & 53,763 & 47,166 & 30,787 & 32,996 & $1,511,906$ \\
\hline & & & & & & & & & & & $4,403,442$ \\
\hline
\end{tabular}


makes it likely that something exceptional has happened. (It is very advisable to have a closer look at these observations.) The robust chain-ladder method will adjust the outliers and calculate the outstanding reserves. The estimated future claims are shown in Table 16, leading to an overall reserve of $R=$ $4,403,442$, which differs significantly from the classical estimate $(18,673,307)$. At this moment it is highly recommended to examine the data and to decide which reserve will be closer to the truth. If the observations of accident year 3 are indeed atypical observations and if there is only a small probability that such exceptionally high claims will occur in the following years, the insurance company will set aside far too much. In that situation it is better to set aside the robust reserve estimate, possibly with a safety margin for the outstanding claims of the third accident year.

Recall that the high observations of the third row will also influence the estimated future claims. In this situation it is useful to know that the data contain some atypical observations. Furthermore there is no reason to believe that the classical chain-ladder method then still produces reliable estimates.

From this section we can conclude that outliers do appear in practice and that the robust chainladder method can handle more than one outlier. It is clear that the robust chain-ladder method is able to detect and adjust these outliers and might be a very convenient aid to construct a more realistic reserve.

\section{Conclusions}

In this article it is illustrated that the outstanding claims reserves by the chain-ladder method are strongly affected by outliers. Often outliers lead to an overestimation of the total reserve estimate, which forces the insurance company to put more aside than actually needed. Depending on the location of the outlier(s), it can also happen that the insurance company underestimates the total reserve estimate (which can lead to bankruptcy in a worst-case scenario).

To solve this problem we propose a robust method that has the ability to calculate the total reserve in such a way that the outlying values are detected and adjusted in the run-off triangle of claims reserves. The detection of outliers is important in practice, because further inspection of these atypical observations can reveal important information. Another approach for obtaining reserve estimates that are less influenced by outliers is by implementing a robust generalized linear model technique.

In addition to the reserve estimates, it is interesting to obtain the standard deviation, which is a measure of dispersion. When it is difficult to estimate a standard error analytically, it is common to adopt the bootstrapping technique. The estimation of the standard error of the robust chain-ladder estimate is calculated with a slightly modified bootstrapping technique (Stromberg 1997). Numerical examples (where we added outliers to the data) show an excellent performance of the robust chainladder method.

From the application of real run-off triangles from a non-life insurance branch in Belgium, it is clear that the proposed robust technique is helpful in gaining insight into the studied claims reserves and the (hidden) outliers and in building up a more realistic reserve. The diagnostic performance of the robust method grows when it is used in addition to the classical approach.

The robust chain-ladder method can easily be implemented and does not need any knowledge of stochastic methods and generalized linear models. All programs are written in the statistical program R.

\section{REFERENCES}

Ashe, F. R. 1986. An Essay at Measuring the Variance of Estimates of Outstanding Claim Payments. ASTIN Bulletin 16S: 99-113.

Barnett, G. AND B. Zehnwirth. 2008. Modeling with the multivariate probabilistic trend family. Casualty Actuarial Society E-Forum, Fall 2008.

Brys, G., M. Hubert, and A. Struyf. 2006. Robust Measures of Tail Weight. Computational Statistics and Data Analysis 50: $733-59$. Cantoni, E., And E. Ronchetti. 2001. Robust Inference for Generalized Linear Models. Journal of the American Statistical Association 96(455): 1022-30.

Carroll, R. J., and S. Pederson. 1993. On Robustness in the Logistic Regression Model. Journal of the Royal Statistical Society, Series B 55: 693-706. 
Croux, G., G. Dhaene, and D. Hoorelbeke. 2003. Robust Standard Errors for Robust Estimators. Research Report, Department of Applied Economics, University of Leuven.

Efron, B., AND R. J. Tibshirani. 1993. An Introduction to the Bootstrap. London: Chapman and Hall.

England, P. D. 2002. Addendum to Analytic and Bootstrap Estimates of Prediction Errors in Claims Reserving. Insurance Mathematics and Economics 31: 461-66.

England, P. D., and R. J. Verrall. 1999. Analytic and Bootstrap Estimates of Prediction Errors in Claims Reserving. Insurance Mathematics and Economics 25: 281-93.

- 2002. Stochastic Claims Reserving in General Insurance. British Actuarial Journal 8(3): 443-544.

- 2006. Predictive Distributions of Outstanding Liabilities in General Insurance. Annals of Actuarial Science 1(2): 221-70.

Hampel, F. R., E. M. Ronchetti, P. J. Rousseeuw, and W. A. Stahel. 1986. Robust Statistics: The Approach Based on Influence Functions. New York: Wiley.

Huber, P. J. 1981. Robust Statistics. New York: Wiley.

Hubert, M., and E. Vandervieren. 2008. An Adjusted Boxplot for Skewed Distributions. Computational Statistics and Data Analysis 52: 5186-5201.

KaAs, R., M. J. Goovaerts, J. Dhaene, and M. Denuit. 2001. Modern Actuarial Risk Theory. Dordrecht: Kluwer.

Künsch, H. R., L. A. Stefanski, AND R. J. Carroll. 1989. Conditionally Unbiased Bounded-Influence Estimation in General Regression Models, with Applications to Generalized Linear Models. Journal of the American Statistical Association 84: 460-66.

Liang, K. Y., ANd S. L. Zeger. 1986. Longitudinal Data Analysis Using Generalized Linear Models. Biometrika 73: 13-22.

Lowe, J. 1994. A Practical Guide to Measuring Reserve Variability Using: Bootstrapping, Operational Time and a Distribution Free Approach. Proceedings of the 1994 General Insurance Convention, Institute of Actuaries and Faculty of Actuaries.

Mack, T. 1993. Distribution-Free Calculation of the Standard Error of Chain-Ladder Reserve Estimates. ASTIN Bulletin 23(2): 21325.

1994. Measuring the Variability of Chain Ladder Reserve Estimates. Casualty Actuarial Society Forum 1: 101-82.

Mack, T., G. Quarg, and C. Braun. 2006. The Mean Square Error of Prediction in the Chain Ladder Reserving Method-A Comment. ASTIN Bulletin 36(2): 543-52.

Mack, T., And G. Venter. 2000. A Comparison of Stochastic Models That Reproduce Chain Ladder Reserve Estimates. Insurance: Mathematics and Economics 26: 101-7.

Maronna, R., D. Martin, and V. Yohai. 2006. Robust Statistics-Theory and Methods. New York: Wiley.

Morgenthaler, S. 1992. Least-Absolute-Deviations Fits for Generalized Linear Models. Biometrika 79: 747-54.

Nelder, J. A., ANd R. W. M. Wedderburn. 1972. Generalized Linear Models. Journal of the Royal Statistical Society 135: 370-84.

Pinheiro, P. J. R., J. M. Andrade e Silva, and M. L. C. Centeno. 2003. Bootstrap Methodology in Claim Reserving. Journal of Risk and Insurance 70(4): 701-14.

Pregibon, D. 1982. Resistant Fits for Some Commonly Used Logistic Models with Medical Applications. Biometrics 55: 574-79.

Preisser, J. S., AND B. F. QAQISH. 1999. Robust Regression for Clustered Data with Applications to Binary Regression. Biometrics 55: $574-79$.

Renshaw, A. E. 1994. On the Second Moment Properties and the Implementation of Certain GLIM Based Stochastic Claims Reserving Models. Actuarial Research Papers no. 65, Department of Actuarial Science and Statistics, City University, London.

Renshaw, A. E., and R. J. Verrall. 1998. A Stochastic Model Underlying the Chain Ladder Technique. British Actuarial Journal 4(4): 903-23.

Rousseeuw, P., AND A. Leroy. 1987. Robust Regression and Outlier Detection. New York: Wiley.

Stefanski, L. A., R. J. Carroll, And D. Ruppert. 1986. Optimally Bounded Score Funetions for Generalized Linear Models with Applications to Logistic Reǵression. Biometrika 73: 413-24.

Stromberg, A. J. 1997. Robust Covariance Estimates Based on Resampling. Journal of Statistical Planning and Inference 57: 321-34. TAYlor, G., AND F. R. Ashe. 1983. Second Moments of Estimates of Outstanding Claims. Journal of Econometrics 23: 37-61.

Tukey, J. W. 1977. Exploratory Data Analysis. Reading, MA: Addison-Wesley.

Van Wouwe, M., T. Verdonck, and K. Van Rompay. 2009. Application of Classical and Robust Chain-ladder Methods: Results for the Belgium Non-Life Business. Global Business and Economics Review, to appear.

Venter, G. 2006. Discussion of the Mean Square Error of Prediction in the Chain-Ladder Reserving Method. ASTIN Bulletin 36(2): 566-71

Verrall, R. 1991. On the Estimation of Reserves from Loglinear Models. Insurance: Mathematics and Economics 10: 75-80.

- 2000. An Investigation into Stochastic Claims Reserving Models and the Chain-Ladder Technique. Insurance: Mathematics and Economics 26: 91-99.

Wüthrich, M. V., M. Merz, And H. Bühlmann. 2008. Bounds on the Estimation Error in the Chain Ladder Method. Scandinavian Actuarial Journal in press.

Discussions on this paper can be submitted until October 1, 2009. The authors reserve the right to reply to any discussion. Please see the Submission Guidelines for Authors on the inside back cover for instructions on the submission of discussions. 